\title{
ON THE ACCEPTANCE OF PROBLEM SOLUTIONS DERIVED FROM INCONSISTENT CONSTRAINTS
}

\begin{abstract}
In this paper, I discuss the main difficulties one encounters when solving problems with inconsistent constraints. I argue that in order to meet these difficulties we need an inconsistency-adaptive logic that enables one to derive as many (interesting) consequences as possible, but that at the same time allows one to determine which consequences can be accepted. I show that the inconsistency-adaptive logic ANA satisfies these requirements.
\end{abstract}

\section{Introduction}

One of the central insights of contemporary philosophy of science is that reasoning in the sciences (as in other domains) is best understood from a problem solving perspective. Within this approach, problems are typically seen as composed of two components: a question to be answered ${ }^{1}$ and a set of constraints (items of information that are relevant for the question in the sense that they provide materials to derive the answer from).$^{2}$ In line with

* Postdoctoral Fellow of the Fund for Scientific Research - Flanders.

${ }^{1}$ In [7], the notion of a problem is conceived in a way that is sufficiently broad to include not only questions to be answered ("intellectual problems") but also states to be realized ("action problems"). In the present paper, the discussion will be restricted to intellectual problems.

2 Some authors use "constraints" in a much broader sense, including also meaning postulates, heuristics, methodological rules, ... (see, for instance, [13] and [11]). However, as these are not relevant for the present paper, I shall not discuss them. 
this, a reasoning process is viewed as a process in which one tries to derive the answer to a question from a set of constraints.

As I argued elsewhere (see [7] and [10]), problem solving in the sciences frequently involves inconsistencies. In nineteenth century thermodynamics, for instance, Rudolf Clausius tried to derive the answer to the question whether Carnot's theorem is valid from two incompatible approaches to thermodynamic phenomena. ${ }^{3}$ Other examples are Planck's and Einstein's derivation of Planck's law (see, for instance, [16]) and Einstein's account of Brownian motion (see, for instance, [13]). ${ }^{4}$ In each of these cases, reasoning from the inconsistencies was seen as necessary to arrive at satisfactory problem solutions. The reason is that the inconsistencies were relevant for the problem at issue, and hence, could not be disregarded. Moreover, at the time the problems were attacked, there were no sufficient grounds to resolve the inconsistencies in a particular way. Hence, simply abandoning some of the constraints in order to restore consistency would have been an arbitrary decision.

Two difficulties are faced in such cases. The first is to determine when a solution of the problem is acceptable. The second is to replace the inconsistent set of constraints by a consistent one. An important guideline for the decision on the first difficulty is that it should be plausible that the solution will remain derivable after the second difficulty has been dismissed (i.e., after the set of inconsistent constraints has been replaced by a consistent alternative).

In [6] and [8], I argue that reasoning processes involving inconsistencies require an inconsistency-adaptive logic. Inconsistency-adaptive logics, originally designed by Diderik Batens (see, for instance, [2] and [3]), have the peculiar property that they 'oscillate' between a paraconsistent 'lower limit logic' and an 'upper limit logic'. The latter is usually Classical Logic henceforth CL. What this comes to is that inconsistency-adaptive logics localize inconsistencies and adapt the rules of inference in view of these: in the neighbourhood of inconsistencies they behave like the lower limit logic; everywhere else they behave like the upper limit logic.

The aim of the present paper is twofold. First, I shall argue that reasoning processes involving inconsistencies require a specific inconsistencyadaptive logic that enables one to derive as many (interesting) consequences as possible, but that at the same time allows one to determine which consequences can be accepted.

\footnotetext{
${ }^{3}$ For a discussion of this example, see [6] and especially [8].

${ }^{4}$ Other interesting case studies on inconsistencies in the sciences can be found in [5], [14] and [15].
} 
Next, I shall show (for the propositional case) that the inconsistencyadaptive logic ANA satisfies these requirements. As I show in [9], ANA contains the entire 'analyzing' fragment of Classical Logic (Disjunctive Syllogism, Modus Tollens, Modus Ponens, Simplification, Negation of the Disjunction, ... ), and moreover validates all classical rules in consistent 'neighbourhoods'. In view of these features, ANA not only leads to an extremely rich consequence set, but moreover allows one to determine, in a very natural way, when a problem solution derived from inconsistent statements should be accepted.

I shall proceed as follows. In section 2, I discuss the main difficulties one encounters when solving problems with inconsistent constraints; I also discuss some central requirements a logic should meet for this type of reasoning process. Next, I briefly present the paraconsistent logic AN and the adaptive logic ANA that is based on it (sections 3 and 4). In section 5, I show that ANA can account for the requirements discussed in section 2. I end with some open problems (section 6).

\section{Reasoning from inconsistencies}

As I mentioned in the introduction, problem solving processes that involve inconsistencies confront one with two difficulties. On the one hand, one should be able to decide when a problem solution is acceptable. On the other hand, one wants to replace the inconsistent set of constraints by a consistent alternative. In this section, I shall argue that a logical analysis of the constraints may play an important role with respect to both difficulties. I shall also argue, however, that this analysis requires a special kind of logic.

Two observations from the history of the sciences are important for the first difficulty. The first is that, when a problem is solved against the background of inconsistent constraints, resolving the inconsistencies involved is not seen as a necessary condition for accepting the solution. Einstein's derivation of Planck's law, for instance, involved several inconsistencies. Still, it was accepted by the scientific community before the inconsistencies were resolved (see [16]). The second observation is that the mere derivability of a solution (by some appropriate paraconsistent logic) is not considered as a sufficient reason for accepting it. A problem solution derived from inconsistent statements is only accepted if it is likely to remain derivable after the inconsistencies are resolved. Planck's and Einstein's derivation of Planck's law form an interesting example here. As Smith convincingly shows in [16], both derivations are structurally similar. As a consequence, paraconsistent 
logics that are rich enough to account for the inferences involved validate both derivations. Still, only Einstein's derivation was accepted. The difference is that in the case of Planck's derivation it was doubtful whether its premises would still be accepted after the inconsistencies were resolved.

Some authors conclude from such examples that the decision whether a problem solution derived from inconsistent statements should be accepted cannot be made on purely formal grounds. According to Smith (see [16]), for instance, this decision depends on the degree of confirmation of the statements involved: only those problem solutions can be accepted that follow from statements with the highest degree of confirmation. The idea behind this is that the more confirming evidence one has for some member of an inconsistent set of statements, the more likely it is that it will be retained in the consistent alternative.

I shall argue, however, that a logical analysis may allow one to distinguish between 'problematic clauses' and 'unproblematic clauses' of a set of constraints, and that this distinction can be important in deciding whether a problem solution derived from inconsistent constraints should be accepted. I first explain what I mean by problematic and unproblematic clauses.

Let $\Gamma^{\dagger}$ be the set of all formulas of the form $B_{1} \vee \cdots \vee B_{n}(n \geq 1$; each $B_{i}$ being an atom) that are derivable from the following set of rules ${ }^{5}$ :

(7) $\&(\Gamma \cup \bigvee(\Delta \cup\{B \& C\})) \approx \&(\Gamma \cup \bigvee((\Delta \cup\{B\}) \cup \bigvee(\Delta \cup\{C\})))$

$$
\bigvee(\&\{A, B\} \cup \Gamma) \vDash \bigvee(\{A\} \cup \Gamma)
$$

where $\&\left\{A_{1}, \ldots, A_{n}\right\}$ denotes any wff obtained by Permutation and Association from $\left(\ldots\left(\left(A_{1} \& A_{i}\right) \& \ldots\right) \& A_{n}\right), \bigvee\left\{A_{1}, \ldots, A_{n}\right\}$ any wff obtained

\footnotetext{
${ }^{5}$ The rules allow one to reduce formulas to conjunctive normal form, and to derive the conjuncts of these. They are formulated in a way that is too complex for $\mathbf{C L}$, but that will make the proofs in section 5 easier.
} 
by Permutation and Association from $\left(\ldots\left(\left(A_{1} \vee A_{i}\right) \vee \ldots\right) \vee A_{n}\right)$, and where $A \approx B$ abbreviates $A \vDash B$ and $B \vDash A$. I say that $A$ is a clause of $\Gamma$ iff $A \in \Gamma^{\dagger}$.

Intuitively, I say that $A$ is an unproblematic clause of $\Gamma$ iff $A \in \Gamma^{\dagger}$ and $\sim A$ can only be obtained from $\Gamma$ by relying on two 'halves' of an inconsistency, or, which comes to the same, can only be obtained from $\Gamma$ by a combination of weakening constructive rules (such as Addition and Irrelevance) and analyzing rules (such as Disjunctive Syllogism and Modus Tollens). Consider, for instance, $\Gamma=\{p, \sim p, r \vee s, \sim r\}$. Here, $\sim s$ can only be obtained from $\Gamma$ by relying on $p$ and $\sim p$, and by applying a weakening constructive rule as well as an analyzing rule. To define the notion of an unproblematic clause more precisely, I have to explain what I mean by analyzing rules.

Analyzing rules typically allow one to break down complex formulas. Some analyzing rules, such as Simplification, Modus Ponens and Disjunctive Syllogism, lead to subformulas of given formulas. Other rules that we consider intuitively as analyzing, such as $A \supset B, \sim B \vDash \sim A$, do not have this property. In that case, however, the formulas involved can be reduced to equivalent formulas with 'roughly' the same degree of complexity in such a way that the resulting conclusion is a subformula of the resulting premises. For instance, $A \supset B$ can be reduced to $\sim A \vee B$, and as $\sim B$ is available, Disjunctive Syllogism can be applied to $\sim A \vee B$ to derive the subformula $\sim A$. In [9], I argue that, at the propositional level, all rules of CL that we consider intuitively as analyzing can be obtained from a set of transformation rules (that equate a complex formula with another formula that has roughly the same degree of complexity) together with two rules that lead to subformulas of given formulas. The rules are (1)-(8) together with

$$
\bigvee(\{A\} \cup \Gamma), \bigvee(\{\sim A\} \cup \Delta) \vDash \bigvee(\Gamma \cup \Delta)
$$

where $\Gamma \cup \Delta \neq \emptyset$.

In view of this result, I say that $A$ is an unproblematic clause of $\Gamma$ iff $A \in \Gamma^{\dagger}$ and $\sim A$ does not follow from $\Gamma$ by (1)-(9). Let me illustrate this with an example. Consider the following set

$$
\{p \& q, \sim r,(p \vee s) \supset r, q \supset t\}
$$

The set of clauses is $\{p, q, \sim r, \sim p \vee r, \sim s \vee r, \sim q \vee t\}$. The unproblematic clauses are $q, \sim s \vee r$, and $\sim q \vee t ; p, \sim r$, and $\sim p \vee r$ are the problematic clauses. 
It is typical for unproblematic clauses that they are not related to the inconsistencies involved: eliminating an unproblematic clause from an inconsistent $\Gamma^{\dagger}$ has no effect on the inconsistencies that follow from $\Gamma^{\dagger}$. Because of this, an important adequacy criterion for consistent alternatives of an inconsistent set of constraints $\Gamma$ is that all unproblematic clauses of $\Gamma$ should be derivable from them. This is rational in view of the fact that, when replacing an inconsistent set of constraints by a consistent alternative, one wants to retain as much information as possible, and hence, one does not want to eliminate more 'parts' of the original set than is necessary for resolving the inconsistencies. In view of this, the fact that one is able to establish that some clause is unproblematic makes it plausible that it will remain derivable from the consistent replacement. So, in some cases problem solutions derived from inconsistent constraints can be accepted on purely formal grounds: it suffices to establish that they follow from unproblematic clauses.

Let us now turn to the second difficulty, namely replacing the inconsistent constraints by a consistent alternative. An important observation here is that problematic clauses are heuristically valuable in this replacement. The reason is that a consistent alternative can never retain all problematic clauses. Hence, the decision that a specific problematic clause should or should not follow from the consistent replacement at once allows one to eliminate some alternatives. Consider (10) again. One of the problematic clauses is $\sim p \vee r$. If one decides that $\sim p \vee r$ should be rejected, one can eliminate all alternatives from which $\sim p \vee r$ follows. If one decides that $\sim p \vee r$ should be accepted, one can eliminate all alternatives from which both $p$ and $\sim r$ follow. In either case, one gains some information on how the consistent replacement should look like. Note that something similar does not hold for unproblematic clauses of $\Gamma$, for instance $\sim s \vee r$. Neither the decision to accept this specific consequence of $\Gamma$ nor the decision to reject it provides information on how the conflict between $p, \sim r$ and $\sim p \vee r$ should be resolved.

So, a logical analysis of the constraints is also here important. It is this analysis that allows one to distinguish between problematic and unproblematic parts, and thus to localize sentences that provide information on how the inconsistencies should be resolved.

The question is now which logic is adequate for this analysis. In view of the previous paragraphs, we can formulate at least three requirements such a logic should meet. The first is that it should enable one to distinguish between problematic and unproblematic clauses. The second is that for unproblematic clauses, it should enable one to solve all those problems that 
eventually (after the inconsistencies are resolved) will be solvable on the basis of CL. (Remember that when replacing an inconsistent set of constraints by a consistent alternative, one wants to retain all unproblematic clauses.) The third requirement is that, for problematic clauses, the logic should be as rich as possible, without leading to triviality. The reason for this is that, in many cases, the decision how an inconsistency should be resolved has to be based on a comparison of the different consistent alternatives. In order for such a comparison to be possible, the logic should be as rich as possible, also for problematic clauses. ${ }^{6}$

In section 5, I shall argue that the inconsistency-adaptive logic ANA meets these requirements. But, first, I have to present ANA. As ANA is an adaptive logic, I shall proceed in two steps. In the next section, I present the lower limit logic AN on which ANA is based; in section 4, I show how ANA can be obtained from AN.

\section{The logic AN}

Let $\mathscr{L}$ be the usual language of $\mathbf{C L}$ in which $\mathrm{S}, \mathrm{C}, \mathrm{V}, \mathrm{P}^{r}$ and $\mathrm{W}$ are, respectively, the set of sentential letters, the set of letters for individual constants, the set of letters for individual variables, the set of letters for predicates of rank $r$, and the set of wffs. Let $\mathscr{L}^{0}$ be the fragment of $\mathscr{L}$ obtained by

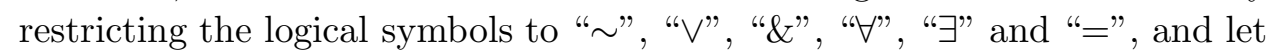
$\mathrm{W}^{0}$ be the set of wffs of $\mathscr{L}^{0}$.

$\mathbf{A N}$ is a two-step system. I first define the three-valued system $\mathbf{A} \mathbf{N}^{\circ}$. The designated values are 1 and $\mathrm{X}$; the non-designated value is 0 . Their intuitive interpretation is as follows:

$$
\begin{aligned}
& v_{M}(A)=0: A \text { is false } \\
& v_{M}(A)=\mathrm{X}: A \text { is contradictory } \\
& v_{M}(A)=1: A \text { is consistently true }
\end{aligned}
$$

An $\mathbf{A} \mathbf{N}^{\circ}$-model is a couple $M=\langle D, v\rangle$ in which $D$ is a set and $v$ is an assignment function. To members of $\mathrm{P}^{r}, v$ assigns a triple $\left\langle\Pi_{1}, \Pi_{\mathrm{X}}, \Pi_{0}\right\rangle$ where

(i) $\Pi_{1} \cup \Pi_{\mathrm{X}} \cup \Pi_{0}=D^{r}$;

(ii) $\quad \Pi_{1} \cap \Pi_{X}=\emptyset ; \Pi_{\mathrm{X}} \cap \Pi_{0}=\emptyset ; \Pi_{1} \cap \Pi_{0}=\emptyset$.

\footnotetext{
${ }^{6}$ In [4], Batens argues that finding a consistent alternative for an inconsistent set of statements can proceed in a more efficient way if, in my terms, only a limited number of problematic consequences is derivable. However, as I argue in [10], the argument is only valid for cases in which the inconsistencies can be resolved in a non-comparative way.
} 
To keep things simple, we stipulate that $v^{1}\left(P^{r}\right)=\Pi_{1}, v^{\mathrm{X}}\left(P^{r}\right)=\Pi_{\mathrm{X}}$, and $v^{0}\left(P^{r}\right)=\Pi_{0}$. The assignment function $v$ is defined as follows:

C1.1

$: S \rightarrow\{0, \mathrm{X}, 1\}$

$\mathrm{C} 1.2 \quad v: \mathrm{C} \cup \mathrm{V} \rightarrow D$

C1.3 $v^{i}: \mathrm{P}^{r} \rightarrow \mathcal{P}\left(D^{r}\right)$

C1.4 $\quad v^{i}:=\rightarrow \mathcal{P}\left(D^{2}\right)$

where $\{\langle\alpha, \alpha\rangle \mid \alpha \in \mathrm{C}\}=v^{1}(=) \cup v^{\mathrm{X}}(=)$

In C1.3-4, the requirement on $v^{0}, v^{\mathrm{X}}$ and $v^{1}$ is as explained; $\mathcal{P}\left(D^{r}\right)$ refers to the power set of the $r$-th Cartesian product of $D$.

The valuation function $v_{M}$ determined by the model $M$ is defined by: ${ }^{7}$

C2.1

C2.2 where $A \in \mathrm{S}, v_{M}(A)=v(A)$

$\mathrm{C} 2.3 \quad v_{M}\left(\pi^{r} \alpha_{1} \ldots \alpha_{r}\right)=i$ iff $\left\langle v\left(\alpha_{1}\right), \ldots, v\left(\alpha_{r}\right)\right\rangle \in v^{i}\left(\pi^{r}\right)$

$\mathrm{C} 2.4 \quad v_{M}(\alpha=\beta)=i$ iff $\langle\alpha, \beta\rangle \in v^{i}(=)$

C2.5-7 clauses for the negation, disjunction and conjunction as defined by the following matrices:

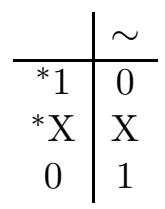

\begin{tabular}{c|ccc}
$\vee$ & 1 & $\mathrm{X}$ & 0 \\
\hline 1 & 1 & 1 & 1 \\
$\mathrm{X}$ & 1 & $\mathrm{X}$ & 0 \\
0 & 1 & 0 & 0
\end{tabular}

\begin{tabular}{c|ccc}
$\&$ & 1 & $\mathrm{X}$ & 0 \\
\hline 1 & 1 & 1 & 0 \\
$\mathrm{X}$ & 1 & $\mathrm{X}$ & 0 \\
0 & 0 & 0 & 0
\end{tabular}

C2.8 $\quad v_{M}((\forall \alpha) A(\alpha))=1$ iff, for all $\beta \in \mathrm{C}, v_{M}(A(\beta))=1$;

$v_{M}((\forall \alpha) A(\alpha))=0$ iff, for some $\beta \in \mathrm{C}, v_{M}(A(\beta))=0$;

otherwise, $v_{M}((\forall \alpha) A(\alpha))=X$

C2.9 $v_{M}((\exists \alpha) A(\alpha))=1$ iff, for some $\beta \in \mathrm{C}, v_{M}(A(\beta))=1$;

$v_{M}((\exists \alpha) A(\alpha))=0$ iff, for all $\beta \in \mathrm{C}, v_{M}(A(\beta))=0$;

otherwise, $v_{M}((\exists \alpha) A(\alpha))=\mathrm{X}$

Truth in a model and semantic consequence are defined as usual.

To obtain $\mathbf{A N}$ from $\mathbf{A} \mathbf{N}^{\circ}$, we first upgrade the latter by the following definitions:

$$
\begin{aligned}
& A \supset B={ }_{\mathrm{df}} \sim A \vee B \\
& A \equiv B={ }_{\mathrm{df}}(A \supset B) \&(B \supset A)
\end{aligned}
$$

\footnotetext{
${ }^{7}$ I only consider $\omega$-complete models.
} 
Next, we specify a procedure for associating each formula with a set of formulas in prenex conjunctive normal form - henceforth PCNF. Where $\mathrm{F}^{a}$ is the set of atoms (primitive formulas and their negations), $A$ is said to be in PCNF iff it has the form

$$
\mathrm{Q} \&\left\{\bigvee \Sigma_{1}, \ldots, \bigvee \Sigma_{n}\right\}
$$

in which $\mathbf{Q}$ is a sequence of quantifiers and $\Sigma_{1} \cup \cdots \cup \Sigma_{n} \subset \mathrm{F}^{a}$. I shall use expressions like $(\dagger)$ to refer to the set of all those wffs as well as (in other contexts) to an arbitrary member of this set. With each $A$ we associate the set $h(A)$ of all formulas that can be obtained from $A$ by applications of D1, $\mathrm{D} 2$, and $\mathrm{T} 1-\mathrm{T} 16$ :

T1 relettering variables (as usual)

T2 $\quad A \vee A \approx A$

T3 $\sim A \approx A$

$\mathrm{T} 4 \quad \sim(A \& B) \approx \sim A \vee \sim B$

T5 $\quad \sim(A \vee B) \approx \sim A \& \sim B$

T6 $\sim(\forall \alpha) A \approx(\exists \alpha) \sim A$

T7 $\sim(\exists \alpha) A \approx(\forall \alpha) \sim A$

T8 $(\forall \alpha) A \vee B \approx(\forall \alpha)(A \vee B)$

T9 $A \vee(\forall \alpha) B \approx(\forall \alpha)(A \vee B)$

$\mathrm{T} 10 \quad(\forall \alpha) A \& B \approx(\forall \alpha)(A \& B)$

T11 $A \&(\forall \alpha) B \approx(\forall \alpha)(A \& B)$

$\mathrm{T} 12 \quad(\exists \alpha) A \vee B \approx(\exists \alpha)(A \vee B)$

$\mathrm{T} 13 \quad A \vee(\exists \alpha) B \approx(\exists \alpha)(A \vee B)$

$\mathrm{T} 14 \quad(\exists \alpha) A \& B \approx(\exists \alpha)(A \& B)$

$\mathrm{T} 15 \quad A \&(\exists \alpha) B \approx(\exists \alpha)(A \& B)$

$\mathrm{T} 16 \quad \&(\Gamma \cup \bigvee(\Delta \cup\{B \& C\})) \approx \&(\Gamma \cup \bigvee(\Delta \cup\{B\}) \cup \bigvee(\Delta \cup\{C\}))$

with the usual restrictions on variables in $\mathrm{T} 8-\mathrm{T} 15$, and with the restriction that T16 may only be applied to entire formulas; T2-15 may be applied to subformulas. Let $g(A)$ be the members of $h(A)$ that are in PCNF.

I now define the logic $\mathbf{A N}$. AN has the same models as $\mathbf{A} \mathbf{N}^{\circ}$, but $\mathbf{A N}$ truth in a model and $\mathbf{A N}$-semantic consequence are defined differently. The 
procedure is somewhat unusual, but, as I show in [9], the resulting logic is well-defined.

Let $f(A)$ be a specific member of $g(A)$, e.g., the one with the lowest Gödel number. Extend the function $f$ to sets as follows: $f(\Gamma)=\{f(A) \mid A \in \Gamma\}$.

Definition. $A$ is AN-true in a model $M$ iff $v_{M}(f(A)) \in\{1, \mathrm{X}\}$.

Definition. $A_{1}, \ldots, A_{n} \vDash_{\mathbf{A N}} B$ iff $B$ is $\mathbf{A N}$-true in all models in which $A_{1}$, $\ldots, A_{n}$ are $\mathbf{A N}$-true.

What this comes to is the following. Wffs in PCNF are AN-true in a model iff they are $\mathbf{A} \mathbf{N}^{\circ}$-true in the model. All other formulas $A$ are associated with their PCNF $f(A)$ and are $\mathbf{A N}$-true in a model iff $f(A)$ is $\mathbf{A N}^{\circ}$-true in the model. Obviously, $\Gamma \vDash_{\mathbf{A N}} A$ iff $f(\Gamma) \vDash_{\mathbf{A N}^{\circ}} f(A)$.

In [9], I show that AN validates (1)-(9), and thus all analyzing rules of CL. I also show that $\mathbf{A N}$ validates all constructive rules that are adjunctive (for instance, $A, B \vDash A \& B$ ). All that is given up are some constructive rules that are weakening, for instance, Addition and Irrelevance, or that are 'paradoxical', for instance, $A \vDash A \vee(B \& \sim B)$ and $A \vDash A \&(B \vee \sim B)$.

\section{The adaptive logic ANA}

The design of the adaptive logic ANA is rather straightforward. Its semantics is obtained by selecting a specific set of $\mathbf{A N}$-models. Let $\mathrm{K}(M)$ stand for the set of primitive formulas $A$ such that $A \& \sim A$ is $\mathbf{A N}$-true (hence $\mathbf{A N ^ { \circ }}$-true) in $M$.

Definition. $M$ is an ANA-model of $\Gamma$ iff (i) $M$ is an AN-model of $\Gamma$ and (ii) there is no $\mathbf{A N}$-model $M^{\prime}$ of $\Gamma$ such that $\mathrm{K}\left(M^{\prime}\right) \subset \mathrm{K}(M)$.

Definition. $\Gamma \vDash_{\text {ANA }} A$ iff $A$ is true in all ANA-models of $\Gamma$.

All classical rules that are validated in $\mathbf{A N}$ are unconditionally valid in ANA. Those that are invalid in AN are conditionally valid in ANA. This means that they apply, provided a specific requirement is satisfied. Where $\Sigma \subset \mathrm{F}^{a}$, let us say that " $\bigvee \Sigma$ behaves consistently with respect to $\Gamma$ " iff $\Gamma \not \nvdash_{\text {AN }} \&\{\bigvee \Sigma, \sim \bigvee \Sigma\}$. It can be shown, for example, that:

$$
\text { if } \Gamma \vDash_{\text {AN }} A \text {, then } \Gamma \vDash_{\text {ANA }} A \vee B
$$

iff each conjunct of $f(A)$ behaves consistently with respect to $\Gamma$; and that

$$
\text { if } \Gamma \vDash_{\text {AN }} B \text {, then } \Gamma \vDash_{\text {ANA }} A \supset B
$$

iff each conjunct of $f(B)$ behaves consistently with respect to $\Gamma$. 


\section{Reasoning from inconsistencies with ANA}

I shall now show that ANA satisfies the conditions discussed in section 2 . I shall restrict the proofs to the propositional fragment of ANA.

The first is that the logic should allow one to distinguish between problematic and unproblematic clauses. Let $\Gamma_{\mathrm{u}}^{\dagger}$ be the set of unproblematic clauses and $\Gamma_{\mathrm{p}}^{\dagger}$ the set of problematic clauses. The richness of ANA allows us to define these sets in a very natural way:

$$
\begin{array}{ll}
\text { Definition. } & \Gamma_{\mathrm{u}}^{\dagger}=\left\{A \mid A \in \Gamma^{\dagger} ; \Gamma \nvdash_{\text {ANA }} \sim A\right\} \\
\text { Definition. } & \Gamma_{\mathrm{p}}^{\dagger}=\left\{A \mid A \in \Gamma^{\dagger} ; \Gamma \vDash_{\text {ANA }} \sim A\right\}
\end{array}
$$

As (1)-(9) are valid in ANA, it is obvious that these definitions capture the intuitive notions introduced in section 2 .

The second condition is that, for unproblematic clauses, the logic should be as rich as CL. As ANA is an inconsistency-adaptive logic with CL as the upper limit logic, $\mathrm{Cn}_{\mathbf{A N A}}(\Gamma)=\mathrm{Cn}_{\mathbf{C L}}(\Gamma)$ for every consistent $\Gamma$. Hence, in order to show that the second requirement holds, I only have to prove that $\Gamma_{\mathrm{u}}^{\dagger}$ is consistent.

Lemma 1. For each $A \in \mathrm{S}, \Gamma_{\mathrm{u}}^{\dagger} \not \nvdash_{\text {ANA }} A$ or $\Gamma_{\mathrm{u}}^{\dagger} \not \nvdash_{\text {ANA }} \sim A$.

Proof. Suppose that $\Gamma_{\mathrm{u}}^{\dagger} \vDash_{\text {ANA }} p$ and $\Gamma_{\mathrm{u}}^{\dagger} \vDash_{\text {ANA }} \sim p$. If $\Gamma_{\mathrm{u}}^{\dagger} \vDash_{\text {ANA }} p$, then (i) $p \in \Gamma_{\mathrm{u}}^{\dagger}$ or (ii) there is a $B$ such that $p \vee B \in \Gamma_{\mathrm{u}}^{\dagger}$ and $\Gamma_{\mathrm{u}}^{\dagger} \vDash_{\text {ANA }} \sim B$. In view of $\Gamma_{\mathrm{u}}^{\dagger} \vDash_{\text {ANA }} \sim p$ and the definition of $\Gamma_{\mathrm{u}}^{\dagger}$, (i) is impossible. If (ii) would hold true, $\Gamma_{\mathrm{u}}^{\dagger} \vDash_{\text {ANA }} \sim p \& \sim B$ and, as (6) is unconditionally valid in ANA, $\Gamma_{\mathrm{u}}^{\dagger} \vDash_{\text {ANA }} \sim(p \vee B)$. But then, $p \vee B \notin \Gamma_{\mathrm{u}}^{\dagger}$.

TheOrem 1. For each $A, \Gamma_{\mathrm{u}}^{\dagger} \not \nvdash_{\text {ANA }} A$ or $\Gamma_{\mathrm{u}}^{\dagger} \not \nvdash_{\text {ANA }} \sim A$.

Proof. In view of Lemma 1 , it is sufficient to prove that, if $\Gamma_{\mathrm{u}}^{\dagger} \vDash_{\text {ANA }} A$ and $\Gamma_{\mathrm{u}}^{\dagger} \vDash_{\text {ANA }} \sim A$, then, for every subformula $B$ of $A, \Gamma_{\mathrm{u}}^{\dagger} \vDash_{\text {ANA }} B$ and $\Gamma_{\mathrm{u}}^{\dagger} \vDash_{\text {ANA }} \sim B$. This is obvious in view of the fact that (1)-(9) are valid in ANA.

The third condition is that, for problematic clauses, the logic should be as rich as possible without leading to triviality. Evidently, the requirement that the logic enables one to derive every sentence that follows by CL from some consistent subset of $\Gamma^{\dagger}$ is too strong. However, a minimal requirement is that, for every maximally consistent subset $\Delta$ of $\Gamma^{\dagger}$, the logic should enable one to derive every atom that follows from $\Delta$ by CL. I shall now show that also this requirement is satisfied by ANA. 
Lemma 2. For all atoms $A$ and all consistent $\Gamma$

$$
\text { if } \Gamma^{\dagger} \vDash_{\mathbf{C L}} A \text {, then } \Gamma^{\dagger} \vDash_{\mathbf{A N}^{\circ}} A \text {. }
$$

Proof. Suppose that $A$ is an atom, that $\Gamma$ is consistent, and that $\Gamma^{\dagger} \not \models \mathbf{A N}^{\circ}$ $A$. Let $\Gamma^{\dagger}=\left\{\bigvee \Sigma_{1}, \ldots, \bigvee \Sigma_{n}\right\}$. As $\Gamma^{\dagger} \not \models \mathbf{A N}^{\circ} A$, there is an $\mathbf{A \mathbf { N } ^ { \circ } \text { -model }}$ $M=\langle v\rangle$, such that $v_{M}\left(\bigvee \Sigma_{i}\right)=\{1, \mathrm{X}\}$, for all $\bigvee \Sigma_{i} \in \Gamma^{\dagger}$, and $v_{M}(A)=0$ Define a CL-model such that, for each atom $B, v^{\prime}(B)=1$ if $v(B) \in\{1, \mathrm{X}\}$, and $v^{\prime}(B)=0$ if $v(B)=0$. Obviously, $v_{M}^{\prime}(A)=0$. Moreover, $v_{M}^{\prime}\left(\bigvee \Sigma_{i}\right)=1$ for all $\bigvee \Sigma_{i} \in \Gamma^{\dagger}$ (by an inspection of the matrix for " $\bigvee$ ").

THEOREM 2. If $\Delta$ is a maximally consistent subset of $\Gamma^{\dagger}$, then $\left\{A \mid \Delta \vDash_{\mathbf{C L}}\right.$ $A ; A$ is an atom $\} \subseteq \mathrm{Cn}_{\text {ANA }}(\Gamma)$.

Proof. Suppose that $A$ is an atom, that $\Delta$ is a maximally consistent subset of $\Gamma^{\dagger}$, and that $\Delta \vDash_{\mathbf{C L}} A$. In that case, there is a finite $\Theta \subseteq \Delta$ such that $\Theta \vDash_{\mathbf{C L}} A$. By Lemma $2, \Theta \vDash_{\mathbf{A N}^{\circ}} A$. But then, $\Theta \vDash_{\mathbf{A N}} A$ (by the definition of $\mathbf{A N}$ ), and thus $\Gamma^{\dagger} \vDash_{\mathbf{A N}} A$. Hence, $\Gamma \vDash_{\mathbf{A N}} A$, (in view of the definition of $\Gamma^{\dagger}$ and the fact that (1)-(9) are valid in $\mathbf{A N}$ ), and $\Gamma \vDash_{\mathbf{A N}} A$ (in view of the fact that, if $\Gamma \vDash_{\text {AN }} A$, then $\Gamma \vDash_{\text {ANA }} A$ ).

\section{Open problems}

In this paper, I showed that (at the propositional level) the inconsistencyadaptive logic ANA enables one to introduce, in a very natural way, a distinction between problematic and unproblematic clauses. This distinction is of central importance for deciding whether problem solutions derived from inconsistent constraints should be accepted. I also showed that for unproblematic clauses, ANA is precisely as rich as CL, and for problematic clauses as rich as possible without leading to triviality.

In a subsequent paper, I shall upgrade the proofs to the predicative level. I shall also formulate definitions for problematic and unproblematic clauses that do not refer to the final analysis of a set of sentences (as is the case for the definitions presented here), but to the stage in a derivation of analysis. The formulation of the definitions in the latter case is made possible by the block-approach of [1]. 


\section{References}

[1] Diderik Batens, "Blocks. The clue to dynamic aspects of logic." Logique $\mathcal{E}^{3}$ Analyse 151-152, 1995, 285-328.

[2] Diderik Batens, "Inconsistency-adaptive logics." In E. Orłowska (ed.), Logic at Work. Essays dedicated to the memory of Helena Rasiowa. Berlin, Springer, 1998, pp. 445-472.

[3] Diderik Batens, "A survey of inconsistency-adaptive logics." Appeared in: D. Batens, C. Mortensen, G. Priest and J.P. Van Bendegem (eds.), Frontiers of Paraconsistent Logic. Baldock, Research Studies Press, 2000, pp. 49-73.

[4] Diderik Batens, "Rich inconsistency-adaptive logics. The clash between heuristic efficiency and realistic reconstruction." Appeared in: F. Beets and E. Gillet (eds.), Logique en perspective. Mélanges offerts à Paul Gochet Brussels, Éditions OUSIA, 2000, pp. 513-543.

[5] Bryson Brown "How to be a realist about inconsistency in science." Studies in History and Philosophy of Science 21, 1990, 281-294.

[6] Joke Meheus, "Adaptive logic in scientific discovery: the case of Clausius." Logique et Analyse 143-144, 1993, 359-391 (appeared in 1996).

[7] Joke Meheus, Wetenschappelijke ontdekking en creativiteit. Een poging tot theorievorming op basis van een conceptuele, methodologische en logische studie. Unpublished doctoral thesis.

[8] Joke Meheus, "Inconsistencies in scientific discovery. Clausius' remarkable derivation of Carnot's theorem." Brepols, in print.

[9] Joke Meheus, "An extremely rich paraconsistent logic and the adaptive logic based on it." Appeared in: D. Batens, C. Mortensen, G. Priest and J. P. Van Bendegem (eds.), Frontiers of Paraconsistent Logic. Baldock, Research Studies Press, 2000, pp. 189-201.

[10] Joke Meheus, "How to reason from inconsistencies." Will appear in: J. Meheus (ed.), Inconsistency in science Kluwer, in print.

[11] Joke Meheus \& Diderik Batens, "Steering problem solving between Cliff Incoherence and Cliff Solitude." Philosophica 58, 1996, 153-187 (appeared in 1998).

[12] Thomas Nickles, "Can Scientific Constraints be violated Rationally?" in T. Nickles (ed.), Scientific Discovery, Logic, and Rationality. Dordrecht, Reidel, 1980, pp. 285-315.

[13] Thomas Nickles, "What is a problem that we may solve it?" Synthese 47, 85-118. 
[14] John D. Norton, "The Logical Inconsistency of the Old Quantum Theory of Black Body Radiation." Philosophy of Science 54, 1987, 327-350.

[15] John D. Norton, "A paradox in Newtonian Gravitation Theory." In PSA 1992 vol. 2, 1993, pp. 412-420.

[16] Joel Smith, "Inconsistency and scientific reasoning." Studies in History and Philosophy of Science 19, 1988, 429-445.

\author{
Joke Meheus \\ Universiteit Gent \\ Centre for Logic and Philosophy of Science \\ Joke.Meheus@rug. ac . be
}

